\title{
Plant and Soil Drivers of Whole-Plant Microbiomes: Variation in Switchgrass Fungi from Coastal to Mountain Sites
}

\author{
Marissa R. Lee and Christine V. Hawkes ${ }^{\dagger}$ \\ Department of Plant and Microbial Biology, North Carolina State University, Raleigh, NC 27607, U.S.A.
}

Accepted for publication 15 October 2020.

\section{ABSTRACT}

Plant-associated microbial diversity is regulated by dispersal from local and regional species pools, as well as filtering by the environment and plant host. However, few studies have simultaneously examined microbial community variation in multiple plant-associated habitats across multiple sites; thus, it is unclear what scales and filters are most important in shaping whole-plant microbiome diversity. To address this, we characterized fungal communities associated with switchgrass (Panicum virgatum $L$.) leaves, roots, and soils within and across 14 stands spanning mountain to coastal ecoregions of North Carolina. Niche differences at small scales (i.e., less than half a kilometer) best explained variation in fungal communities. However, the specific environmental drivers of fungal community composition differed for leaves, roots, and soils. Leaf and root fungi were both affected by plant height, whereas soil fungi were controlled by stand age. Different soil properties were important for fungi in all plant-associated habitats: $\mathrm{K}, \mathrm{P}$, and $\mathrm{pH}$ for leaves; clay, $\mathrm{Mn}$, and $\mathrm{pH}$ for roots; and clay, dissolved organic carbon, total inorganic $\mathrm{N}$, and $\mathrm{Cu}$ for soils. Climate and spatial variables were not significant, further supporting the key role of plant and soil properties. Advances such as these will help us explain, predict, and manipulate microbial assemblages that support plant growth in managed and natural systems.

Keywords: community assembly, distance-decay, ecology, environmental filtering, fungi, leaf, microbiome, mycology, Panicum virgatum, rhizosphere and phyllosphere, root, soil, spatial scaling
The phytobiome represents a growing but largely untapped resource for plant management, particularly the microbiome component. To develop microbiome tools in managed ecosystems, we must understand the mechanisms underlying assembly of plant microbiome communities across landscapes. Ecological theory suggests that microbiome assembly depends on a combination of

${ }^{\dagger}$ Corresponding author: C. V. Hawkes; chawkes@ncsu.edu

Funding: This work was supported by a Department of Energy Genomic Sciences Program SFA (SCW1039) subaward from Lawrence Livermore National Lab, USDA Hatch (accession no. 1018688), and startup funding from North Carolina State University to C. V. Hawkes.

Author contributions: The experiment was designed by C.V.H., M.R.L. collected and analyzed the data, and M.R.L. and C.V.H. wrote the manuscript.

*The $\boldsymbol{e}$-Xtra logo stands for "electronic extra" and indicates that supplementary materials are published online.

The author(s) declare no conflict of interest.

(C) 2021 The American Phytopathological Society stochastic dispersal from local and regional species pools, as well as filtering by the local environment and the plant host (Chesson 2000; Tilman 2004). Understanding the importance of stochastic dispersal effects versus environmental filtering also speaks to the ongoing debate on whether neutral or niche processes control community assembly (Büchi and Vuilleumier 2014; Zhou and Ning 2017). Because few studies address the whole-plant microbiome across multiple sites, we still do not know how these processes vary across both spatial scales and plant-associated microbial habitats such as leaves, roots, and soils. For example, it isn't clear whether the "local" neighborhood is the same for aboveground versus belowground plant tissues. This type of information will facilitate development of microbiomes as reliable tools in bioenergy crops and other agricultural ecosystems, where a key challenge to regulating the microbiome lies in understanding and harnessing drivers of spatial and temporal heterogeneity.

In microbial communities, stochasticity is assumed to arise largely from random patterns of dispersal. Some studies have shown well-mixed and consistent dispersal pools (Gusareva et al. 2019; Kivlin et al. 2014), whereas others demonstrate strong distance- 
decay patterns driven by dispersal limitation (Bell 2010; Cline and Zak 2013; Peay et al. 2010). However, there is substantial variation in the presence and strength of distance-decay relationships (Fan et al. 2017; Queloz et al. 2010; Vacher et al. 2016). The lack of consistent results may, in part, reflect different microbial habitats such as plant leaves and roots where the contribution of aerial dispersal to assembly may vary. In root symbionts, for instance, only a subset of arbuscular mycorrhizal taxa with small spores are common in aerial pools (Chaudhary et al. 2020) and seedlings are often colonized by existing mycorrhizal networks (Van der Heijden 2004). An additional problem with inferring dispersal limitation from spatial constraints is that selection based on environmental heterogeneity can affect the slope of the distance-decay curve, meaning that space and environment must be decoupled.

Postdispersal, the environment acts as a filter on establishment, resulting in patterns of species sorting according to their abiotic and biotic tolerances. Although environmental sorting appears to be a common controller of microbial community assembly (Hanson et al. 2012), the specific aspects of the environment that matter most are unlikely to be the same across plant-associated habitats. Plant microbiomes effectively have two filters-host attributes and environmental conditions - that will constrain microbiome community assembly. Host leaf and root tissues vary in resource availability (Craine et al. 2005) and the extent to which these habitats buffer against environmental stress (Thomas et al. 2016), likely with consequences for the growth and survival of microorganisms. Microbial taxa that reside in leaf tissue must depend on the plant for nutrition, whereas many belowground microorganisms can also access nutrients in soil organic matter (Lindahl and Tunlid 2014). Variation in soil conditions, especially $\mathrm{pH}$, can shape soil and root-associated microbial communities (Vos et al. 2013), whereas climate conditions are often important for leaf endophytes (Bálint et al. 2014; Giauque and Hawkes 2013, 2016). However, to identify the factors that are most important for plant-associated microorganisms while controlling for spatial variation requires sampling within and across a range of sites that vary in environmental conditions.

To address drivers of variation in the plant microbiome, we focused on fungal communities associated with switchgrass (Panicum virgatum L.) leaves, roots, and soils within and across 14 stands spanning the lower mountains, piedmont, and coastal plain of North Carolina, United States. We selected switchgrass because it is a native, long-lived perennial that is likely to develop both spatial and temporal variation in the microbiome. As a potential cellulosic bioenergy crop, it is also widely planted in North Carolina, allowing for assessment of sites throughout the state, which vary widely in site and environmental characteristics such as soil properties. Other studies have shown that strong climate gradients can affect switchgrass-associated fungi (Giauque and Hawkes 2013) but little work has been done across other environmental gradients. Finally, because switchgrass-associated fungi are important mediators of host physiology (Giauque and Hawkes 2013; Kleczewski et al. 2012) and, therefore, likely candidates for future manipulation, our findings will be pertinent for setting boundaries on the spatial scales at which switchgrass fungal community assembly mechanisms are shared.

Overall, we expected that fungi in switchgrass leaves, roots, and soils would differ at both individual and landscape scales. More specifically, we expected leaf fungi to be unique compared with root and soil fungi. Although the majority of foliar bacteria in $P$. virgatum at a single site were recently identified as a subset of local soils (Grady et al. 2019), root fungi may reflect soils more so than leaves because the latter have greater potential for being influenced by aerial dispersal. We further hypothesized that aerial dispersal would lead to foliar fungal communities that were more similar among plants and decayed more slowly with distance than those in roots or soils, given the known small-scale heterogeneity in soil microbial communities (Vos et al. 2013). Because of the presumed dependence of plant-associated fungi on plant carbon supply (McCutcheon and Lekberg 2019), we further expected plant size to have a greater influence on leaf and root communities. In contrast, soil properties should be more important for belowground communities immersed in and buffered by the soil matrix, whereas leaves should be more strongly affected by climate conditions.

\section{MATERIALS AND METHODS}

Experimental design. We selected 14 sites where switchgrass was grown across the lower mountains, piedmont, and coastal plain of North Carolina (462-km span). Sites were chosen to maximize variation in stand age and environmental conditions (Supplementary Table S1). Between late September and early October 2018, we visited the sites to sample plant tissues and soils.

Eight plants per site were sampled for leaf, root, and soil material in a stratified random design as follows, using random points along eight equally spaced transects across the length of the stand. For sites that included more than one contiguous rectangular plant stand, the eight transects were distributed across stands so as to keep the distances between transects in a contiguous stand relatively constant. Five leaves with no visible signs of damage or disease were collected from throughout the canopy of each target plant. Roots and soils were collected to $15 \mathrm{~cm}$ in depth directly adjacent to the plant base on its north and south sides. All samples were stored on ice for transport to the lab. In the lab, fine roots $(<2 \mathrm{~mm})$ were separated from soils, washed to remove soil residue, and frozen for DNA extractions. Soil, leaf, and root aliquots were also frozen at $-80^{\circ} \mathrm{C}$ for DNA extraction. Additional fresh and air-dried soil aliquots were used in biogeochemical extractions and analysis of soil physical and chemical properties.

Plant, soil, and climate characteristics. For each plant, we collected the following data: GPS location, mean maximum height of the three tallest, fully extended tillers (in meters), and maximum basal width and the width perpendicular to the maximum (in meters). Plant-specific GPS locations were measured or calculated based on the stand perimeter and transect dimensions to compute pairwise spatial distances between samples (1.2 $\mathrm{m}$ to $462 \mathrm{~km}$ ). Basal widths were used to estimate basal area (in square meters) as an ellipse. Stand age was based on the land manager's site records.

We measured soil chemical and physical properties for soils associated with each plant sample. Soil $\mathrm{pH}$ was measured by mixing soil and distilled water in a 1:1 ratio. For total inorganic $\mathrm{N}$ (TIN), fresh soils were extracted with $2 \mathrm{M} \mathrm{KCl}$ in a 1:3 ratio and $\mathrm{NH}_{4}^{+}$and $\mathrm{NO}_{3}^{-}$were quantified colorimetrically (Doane and Horwáth 2003; Mulvaney 1996). To measure dissolved organic carbon (DOC), soils were extracted in $0.5 \mathrm{M} \mathrm{K}_{2} \mathrm{SO}_{4}$ in a 1:4 ratio. For microbial biomass carbon (MBC), soils were fumigated with chloroform and then extracted in $\mathrm{K}_{2} \mathrm{SO}_{4}$ (Scott-Denton et al. 2006; Vance et al. 1987). In both fumigated and unfumigated extracts, $C$ was quantified colorimetrically (Bartlett and Ross 1988). MBC was calculated as the difference between fumigated and unfumigated extractions divided by 0.45 to convert from salt-extractable to total $\mathrm{C}$ (Vance et al. 1987). Extractable $\mathrm{PO}_{4}^{-}$was measured by incubating soils with positively charged ion exchange resin strips, extracting strips with $0.5 \mathrm{M} \mathrm{HCl}$, and then quantifying colorimetrically (Robertson et al. 1999). Soil texture (percent sand and clay) was determined via hydrometer method (Bouyoucos 1962). Soil C and $\mathrm{N}$ (percent) were measured by combustion at the Environmental and Agricultural Testing Service at North Carolina State University. 
Soil elemental concentrations of $\mathrm{P}, \mathrm{K}, \mathrm{S}, \mathrm{Ca}, \mathrm{Cu}, \mathrm{Mg}, \mathrm{Mn}$, and $\mathrm{Zn}$; bulk density; and soil organic matter were measured by the North Carolina Department of Agriculture and Consumer Services. Mean annual precipitation (MAP) and temperature (MAT) based on 30year normals (1981 to 2010) were obtained at the site level (PRISM Climate Group; https://prism.oregonstate.edu/explorer).

Amplicon metagenomics. Fungal DNA was extracted from 40 to $50 \mathrm{mg}$ of leaf and root material using the Synergy 2.0 Plant DNA Extraction Kit (OPS Diagnostics, Lebanon, NJ, U.S.A.) and from $250 \mathrm{mg}$ of soil material using the DNeasy PowerSoil Kit (Qiagen, Germantown, MD, U.S.A.). Amplicons to identify fungal taxa were generated using the primer set 5.8S_FUN and ITS4_FUN (Taylor et al. 2016) with Illumina adapters. We also included a custom peptide nucleic acid (PNA) sequence to block nontarget amplification of host DNA (Supplementary Information Methods and Results; Supplementary Figs. S1 and S2). For root and leaf samples, the following PCR conditions were used to generate fungal amplicons: $12.5 \mu \mathrm{l}$ of $2 \times$ KAPA Taq Ready Mix (Roche, Pleasanton, CA, U.S.A.), $0.625 \mu \mathrm{l}$ of bovine serum albumin at $20 \mathrm{mg} / \mathrm{ml}$, $1 \mu \mathrm{l}$ of $5 \mathrm{mM}$ forward primer, $1 \mu \mathrm{l}$ of $5 \mathrm{mM}$ reverse primer, $2.5 \mu \mathrm{l}$ of $15 \mu \mathrm{M}$ PNA, $25 \mathrm{ng}$ of DNA template, and $2.375 \mu \mathrm{l}$ of water. For soils, PNA was replaced with an equal volume of water. Amplicons were purified of fragments $<50 \mathrm{bp}$ using Agencourt AMpure XP beads with a sample/bead ratio of 1:1.8 (Beckman Coulter, Indianapolis, IN, U.S.A.). Genomic libraries were prepared using the Nextera XT Index Kit (Illumina, San Diego, CA, U.S.A.). Pairedend $(2 \times 250)$ sequencing was performed on Illumina MiSeq v2 in the Genomic Sciences Laboratory at North Carolina State University (Raleigh, NC, U.S.A.). All sequences were deposited in the NCBI Short Read Archive under BioProject PRJNA648664.

Bioinformatics. To identify amplicon sequence variants (ASVs), we used the dada2 bioinformatic pipeline ( $\mathrm{R}$ package dada2, v1.10.1) (Callahan et al. 2016) with post clustering curation (R package lulu, v0.1.0, Frøslev et al. 2017). We removed ASVs with fewer than six occurrences, which was the number of occurrences needed to maximize variation explained by the first double principal coordinate analysis (DPCoA) axis without requiring an ASV to be present in all samples from a site (eight samples per site). ASV taxonomic assignments were made using the UNITE database (Nilsson et al. 2018) and the dada2 function "assignTaxonomy" (Wang et al. 2016). For fungal ASVs that were assigned to a phylum, additional classification approaches were employed: RDP classifier using the Warcup fungal internal transcribed spacer (ITS) database (Deshpande et al. 2017; Wang et al. 2016) and pairwise sequence alignment in MycoBank (Robert et al. 2013). Ultimately, taxonomic identities could be assigned to $100 \%$ of ASVs at the phylum level, $84.3 \%$ at class, $79.4 \%$ at order, $62.9 \%$ to family, and $41.4 \%$ at genus. After removal of nonfungal ASVs, there were 932 ASVs identified. Sequencing depth provided adequate coverage in all plant-associated habitats (Supplementary Fig. S3). A phylogenetic tree was built using taxonomic assignments and the perl script "taxonomy_to_tree.pl" provided by Tedersoo et al. (2018). The script maps the ITS-based taxonomic assignments to a Newickformatted hierarchical framework with an equal branch length for each taxonomic rank that can be used as a phylogenetic tree to test evolutionary ecology hypotheses. This approach was taken because ITS region sequences are too variable to infer relatedness among distantly related taxa using traditional phylogenetic methods (Gao et al. 2020; Tedersoo et al. 2018). The ASV, sample data, taxonomy, and tree matrices are available at https:/github.com/ HawkesLab/Lee_Hawkes_PhytobiomesJ.

Statistical analyses. Our overall approach was to examine how switchgrass leaf, root, and soil varied in (i) fungal $\alpha$ and $\beta$ diversity, (ii) distance-decay relationships, (iii) relationships of fungal community composition to environmental and spatial variables, and (iv) shifts in ASVs with key environmental variables.

All statistics were run in $\mathrm{R}$ v3.5.3 ( $\mathrm{R}$ Core Team 2014). All analyses were conducted both with and without consideration of phylogenetic differences in fungal communities except for the structural equation models (SEMs). SEMs were only run with phylogenetically weighted data, because only a small fraction of variance was explained in the taxonomic data by the first principal component (leaf: $9.4 \%$, root: $5.2 \%$, and soil: $6.0 \%$ ). Otherwise, results from both phylogenetically weighted and unweighted metrics were largely the same; therefore, we focus here on analyses with phylogenetic information. Additional details regarding taxonomic analyses can found in the Supplementary Information.

To test for differences in $\alpha$ diversity between plant-associated habitats, ASV richness and Faith's phylogenetic diversity were calculated and tested using analysis of variance with site as a random effect and Tukey posthoc comparisons ( $\mathrm{R}$ package picante::pd, v1.8.1) (Kembel et al. 2010). We used ASV occupancy in plant samples to examine how ASVs were distributed across the range of sites in each plant-associated habitat.

To examine whether fungal communities differed based on plantassociated habitat (i.e., leaf, root, or soil), site, or their interaction, we performed multivariate analysis of variance using residual randomization in permutation procedures (R package RRPP:: lm.rrpp, v0.5.2) (Collyer and Adams 2018). The fungal community was represented by phylogenetically weighted community distances extracted from a DPCoA that included the ASV matrix and the square root cophenetic phylogenetic distances between ASVs (R package ade4::dpcoa, v1.7-13) (Pavoine et al. 2004). In the case of a significant interaction, posthoc tests were conducted to determine the significance of site for each plant-associated habitat.

To examine decay in the relationship between pairwise community similarity and spatial distance, we estimated breakpoint locations for each plant-associated habitat given a linear model and number of breaks. Pairwise community similarities were calculated using the PhySor Index, which is a phylogenetic version of BrayCurtis similarity ( $\mathrm{R}$ package picante::phylosor, v1.8.1) (Bryant et al. 2008). Euclidean distances between samples were calculated from GPS coordinates. One breakpoint was estimated per plant-associated habitat type using iterative model fitting in the $\mathrm{R}$ package segmented, v1.1 (Muggeo 2003). To test for nonzero differences in slope before and after the breakpoint, Davies tests were used (segmented::davies.test) (Davies 2002). The model was split into two linear regressions separated by the mean overall breakpoint and we used analysis of covariance to examine intercept and slope differences between plant-associated habitats with Tukey posthocs and least square mean $t$ tests.

To identify the drivers of leaf, root, and soil fungal communities within and across sites, we used SEM. In each SEM, fungal community composition was represented by the first DPCoA axis based on phylogenetically weighted community composition as described above but calculated separately for roots, leaves, and soils based on the significant interaction term in the $R R P P$ tests described above.

Potential predictors in the SEM included environmental and spatial variables but final variables were chosen based on minimizing correlations and model selection. When predictor variables were highly correlated (Pearson's $r>10.80 \mathrm{l}$ ), only one was retained for modeling, resulting in 20 predictor variables representing variation in soil properties, stand age, plant size, climate, and space (Supplementary Tables S2 and S3). Because climate and spatial variables were correlated (latitude and MAP, $r=-0.78$; longitude and MAT, $r=0.82$ ), we ran two different models based on the inclusion of environmental variables with either climate or spatial variables. 
To determine which of the remaining variables to include in each SEM, model selection was performed by fitting a generalized linear model with all variables as predictors using a lasso penalty chosen based on the minimum mean cross-validated error ( $\mathrm{R}$ package glmnet, v2.0-18) (Friedman et al. 2010). Prior to model selection, the following predictor variables were transformed to improve normality: (i) log-transformed variables included plant basal area, stand age, moisture, DOC, $\mathrm{PO}_{4}^{-}, \mathrm{TIN}, \mathrm{Cu}, \mathrm{K}, \mathrm{Mg}, \mathrm{Mn}, \mathrm{P}, \mathrm{Zn}$, and MAT and (ii) logit-transformed variables included percent soil $\mathrm{C}$, clay, and sand. The selection process with either climate or spatial variables retained 12 to 16 predictor variables per plant-associated habitat type (Supplementary Table S3). To facilitate SEM fitting, all variables were divided by the absolute value of their range to equalize variances (Mamet et al. 2017).

An initial SEM was constructed based on the following assumptions about predictor-to-predictor relationships: (i) soil texture influences soil resources and plant size, (ii) plant stand age influences soil resources and plant size, and (iii) climate influences plant size (R package lavaan::sem, v0.6-5) (Rosseel 2012). In addition, paths specifying the influence of each predictor on fungal composition were included. Covariance relationships among variables in the same category (e.g., soil resources) were added if the modification index value exceeded 10 . Then, constraints were progressively released to improve model fit, indicated by reduced $\chi^{2}$ and Akaike Information Criterion (AIC) (Mamet et al. 2017). In other words, nonsignificant paths $(P>0.05)$ and poorly estimated relationships were iteratively removed. The resulting best model was chosen based on AIC, comparative fit index, and root mean square error of approximation. Note that soil DPCoA1 has been multiplied by -1 to facilitate interpretation across plant-associated habitat communities. The relative explanatory power of variables identified as having direct effects on fungal composition in the SEMs were further investigated with multiple regression.

To identify associations between specific ASVs and key environmental gradients identified in final SEMs, we fitted joint species distribution models using hierarchical models of species communities (R package HMSC, v3.0.4) (Tikhonov et al. 2020). Environmental predictors included all significant direct effects in the final SEM models and total library size to account for variation in sampling effort. Both ASV abundance and presence or absence were analyzed. For ASV abundance, the matrix of ASV reads was $\log$ transformed and modeled with a Gaussian distribution. For ASV presence, the ASV read matrix was converted to binary and modeled with Probit regression. In all models, phylogenetic covariance was included as a random effect on the estimated ASV-environment slope parameters $(\beta)$ to account for correlation in ASV-environment relationships due to evolutionary history. The influence of phylogeny, represented by the parameter $\rho$, is reported for each model. Parameter $\rho$ ranges from 0 to 1 with 0 signifying no influence of phylogeny on ASV-environment relationships. Parameters were estimated through Monte Carlo Markov chain (MCMC) sampling using 1,000 Gibbs steps and a burn-in of 500 . Model convergence was determined based on visual inspection of MCMC traces and potential scale reduction factors close to 1 across all parameter estimates. Significant ASV-environment correlations were identified for abundance models based on whether the $95 \%$ confidence intervals (CIs) of the slope estimate overlapped with 0 . For presence-or-absence models, the confidence threshold was increased to $99 \%$ to simplify summary figures and tables.

\section{RESULTS}

Fungal community diversity and composition. Soil fungal communities were more taxa rich and phylogenetically diverse than leaf or root communities (Supplementary Fig. S4; Supplementary Table S4) associated with switchgrass. Leaf ASVs tended to be more cosmopolitan than root and soil ASVs, with $8 \%$ of leaf ASVs $(n=18)$ found in at least half of all samples compared with $0.01 \%$ for root ASVs $(n=1)$ and $0.01 \%$ for soils $(n=4)$ (Supplementary Fig. S5). The majority of ASVs detected (approximately 65\%) were shared between roots and soils (Fig. 1A). Among the 675 ASVs found in roots, only approximately $2 \%$ were unique to roots and, among the 789 soil ASVs, $14 \%$ were only found in soil. In contrast, $57 \%$ of the 207 ASVs found in leaves were unique to leaves. Ascomycota, Basidiomycota, Glomeromycota, and Chytridiomycota comprised 30,27,27, and 8\%, respectively, of all taxa in the dataset. The remainder consisted of Rozellomycota, Mortierellomycota, Mucoromycota, Blastocladiomycota, Basidiobolomycota, Entorrhizomycota, Kickxellomycota, and Zoopagomycota.

Fungal community composition significantly differed based on plant-associated habitat (Fig. 1B) but the differences among leaf, root, and soil fungi depended on site (Supplementary Tables S5 and S6). Similarly, results based on taxonomic community composition showed a significant interaction between site and plant-associated habitat (Supplementary Information).

Distance-decay relationships. The relationship between fungal community similarity and spatial distance was not linear (Fig. 2; Supplementary Fig. S6; Supplementary Tables S7 and S8). Communities became more dissimilar the further apart they were only up to approximately $0.5 \mathrm{~km}$, beyond which community similarities were relatively constant. Leaf communities maintained a higher degree of similarity across all distances compared with root and soil fungi (Fig. 2; Supplementary Table S7). A slightly shorter breakpoint was found for leaf $(0.31 \mathrm{~km} \pm 0.0595 \% \mathrm{CI})$ than for soil $(0.47 \mathrm{~km} \pm 0.0595 \% \mathrm{CI})$ fungal community distance-decay relationships, with roots intermediate $(0.38 \mathrm{~km} \pm 0.0595 \% \mathrm{CI})$ (Supplementary Table S5).

Decay rates (slopes) did not differ by plant-associated habitat prior to the overall mean breakpoint (Fig. 2; Supplementary Table S7). After the overall mean breakpoint, leaf community decay rates were slightly but significantly faster than root and soil communities $(P<0.001)$ (Supplementary Table S7).

Influence of soil properties, plant size, stand age, climate, and location. The best-fit SEMs for leaf, root, and soil fungi all included plant traits, soil texture, and soil resources (Fig. 3; Supplementary Table S9). Climate and spatial variables were not present in any final SEMs because variation in fungal communities was more parsimoniously captured by other variables. The proportion of variance explained in fungal composition by SEM predictors was lowest for roots $\left(R^{2}=0.16\right)$ and almost double that for leaves $\left(R^{2}=\right.$ $0.29)$ and soils $\left(R^{2}=0.28\right)$ (Supplementary Table S9). The majority of variance was explained by variables with direct effects on fungal composition (Fig. 3; Supplementary Table S10). Joint species distribution models performed approximately equally well across communities, both when ASV presence (area under the receiver operating characteristic curve $=0.79$ to 0.82$)$ and abundance $\left(R^{2}=\right.$ 0.20 to 0.25 ) data were used (Fig. 4; Supplementary Table S11). Phylogenetic relatedness played a large role when ASV abundance was analyzed but had a much smaller role in explaining ASV presence, as shown by $\rho$ estimate differences (Supplementary Fig. S7).

The following conditions supported leaf fungal communities with relatively more Ascomycota and fewer Basidiomycota: higher soil K (partial $r^{2}=0.12$ ), lower soil P (partial $r^{2}=0.10$ ), shorter plants (partial $r^{2}=0.05$ ), and more neutral soil $\mathrm{pH}\left(\right.$ partial $r^{2}=0.05$ ) (Fig. 3A; Supplementary Tables S9 and S10; Supplementary Fig. $\mathrm{S} 8$ ). In addition, sand and $\mathrm{pH}$ had indirect effects on fungal composition: higher sand content was correlated with more soil $\mathrm{P}$ 
(Fig. 3A; Supplementary Table S9) and higher soil pH was correlated with taller plants (Fig. 3A; Supplementary Table S9). The majority of significant ASV-environment relationships involved Ascomycetes from the class Dothideomycetes, including many from known endophytic genera (Fig. 4; Supplementary Fig. S9; Supplementary Tables S12 and S13). Basidiomycete shifts were primarily due to Puccinia emaculata (switchgrass rust) and yeasts in the order Tremellales (Fig. 4; Supplementary Fig. S9; Supplementary Table S12 and S13).

In roots, a shift to relatively more members of the phyla Ascomycota and Glomeromycota and fewer Basidiomycota was supported by higher clay content (partial $r^{2}=0.08$ ), lower soil Mn (partial $r^{2}=0.07$ ), more neutral $\mathrm{pH}$ (partial $r^{2}=0.05$ ), and shorter plants (partial $r^{2}=0.04$ ) (Fig. 3B; Supplementary Tables S9 and S10; Supplementary Fig. S8). Indirect effects were also evident, with higher soil sand content correlated with lower Mn and higher $\mathrm{pH}$, as well as taller plants associated with higher soil $\mathrm{pH}$ and lower Mn (Fig. 3B; Supplementary Table S9). In moving from lower to higher clay soils, root mycorrhizal taxa shifted from predominantly Glomus spp. to Kamienskia spp. and several members of the order Sebacinales (Fig. 4; Supplementary Table S12). Many rootassociated ASVs were more likely to be present in higher Mn conditions ( $>30 \mathrm{ASVs}$ ), whereas a small subset of Ascomycota, including the dark septate endophyte Acidomelania panicicola, were more likely to be found in low Mn conditions (Fig. 4; Supplementary Table S12). Basidiomycetes in the classes Agaricomycetes and Tremellomycetes were less likely to be found in roots growing in neutral pH soils (Fig. 4; Supplementary Tables S12 and S13).

Soil fungal communities with relatively more members of Ascomycota and Glomeromycota and fewer Basidiomycota were associated with younger stands (partial $r^{2}=0.18$ ), lower DOC (partial $r^{2}=0.22$ ), higher TIN (partial $r^{2}=0.10$ ), higher clay content (partial $r^{2}=0.08$ ), and higher $\mathrm{Cu}$ (partial $r^{2}=0.07$ ) (Fig. 3C; Supplementary Tables S9 and S10; Supplementary Fig. S8). In addition, older stands had more soil $\mathrm{Cu}$ and TIN, whereas higher clay soils had lower TIN and more DOC (Fig. 3C; Supplementary Table S9). In older switchgrass stands, approximately $40 \%$ of all Glomeromycota ASVs significantly decreased in abundance (108 of 253 ASVs) (Supplementary Fig. S9; Supplementary Table S13). Most fungi that were responsive to DOC declined or were absent in high DOC soils, with only one Basidiomycete yeast significantly associated with higher DOC (Fig. 4; Supplementary Fig. S9;
Supplementary Tables S12 and S13). Soils with more clay were associated with diverse Ascomycota, as well as the presence of a small number of mycorrhizal ASVs in the phylum Glomeromycota and order Sebacinales, a subset of Agaricomycetes, and a number of chytrids (Supplementary Fig. S9; Supplementary Table S12). Higher TIN soils were associated with increases in only three ASVs (two chytrids and one Metarhizium sp.) (Supplementary Fig. S9; Supplementary Table S13).

\section{DISCUSSION}

Leaf, root, and soil fungi associated with switchgrass responded to environmental gradients across scales from individual plants to landscapes. At the individual level, plant leaves harbored unique fungal communities compared with roots and soils. Across the landscape, there was significant variation in both the distributions and drivers of leaf, root, and soil fungal communities. Distancedecay patterns suggest strong dispersal limitation or environmental heterogeneity and sorting in switchgrass-associated fungi. The drivers of these patterns were soil and plant properties, not climate or spatial factors, further supporting a role for local environmental sorting. The fundamental differences between switchgrass leaf, root, and soil fungi and their environmental dependencies demonstrates the complexity of plant microbiome community assembly and supports the need to understand the entire plant microbiome at landscape scales.

Consistent with our expectations, switchgrass leaf fungal communities were substantially different from root and soil fungi. Fungal ASVs unique to leaves are likely habitat specialists, given that sampling was adequate based on rarefaction curves. In previous culture-based characterization of switchgrass leaf and root fungi, only $20 \%$ of fungal taxa were shared between plant tissues (Ghimire et al. 2010). This pattern is consistent across studies of other plant species in diverse habitats, where 11 to $21 \%$ of taxa were found in both leaf and belowground root or soil habitats (Martins et al. 2016; Qian et al. 2019; Sánchez Márquez et al. 2010). Nevertheless, this result contrasts with the recent finding that leaf-associated bacterial communities in switchgrass and Miscanthus $\times$ giganteus are a nested subset of those found in soils (Grady et al. 2019). One possibility is that fungi colonizing switchgrass leaves may predominantly come from other leaves, whereas bacteria may disperse from nearby soil. A recent study of Panicum hallii across a rain gradient supports this idea, with leaves of nearby plant species
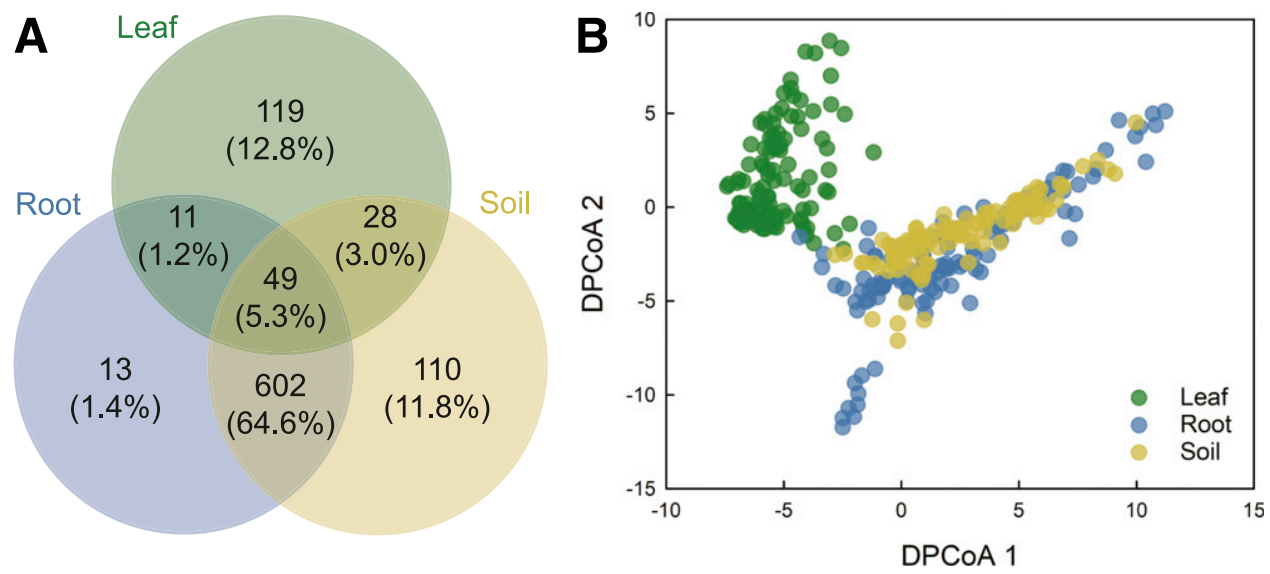

Fig. 1. Fungal community diversity and composition in switchgrass leaves, roots, and soils. A, Venn diagram of fungal amplicon sequence variants (ASVs) that are shared and unique to plant-associated habitat types. Percentages are calculated relative to all ASVs $(n=932)$ found in all habitats. B, Ordination of fungal communities based on phylogenetically weighted community distances using double principal coordinate analysis (DPCoA). 
serving as the primary source of foliar fungi and not soils (C. V. Hawkes, unpublished data).

Although leaf, root, and soil fungi differed, their distance-decay patterns were very similar. We expected leaf fungi to have flatter distance-decay slopes due to the likelihood of higher dispersal rates but, at short distances $(<0.5 \mathrm{~km})$, decay was rapid and the rate of decay was the same across leaves, roots, and soils. On average, fungal community similarities decayed with distances within and between sites that ranged in size from 0.03 to $34 \mathrm{~km}^{2}$ (median $1.5 \mathrm{~km}^{2}$ ). Similarly, rapid decay at short distances has been found in

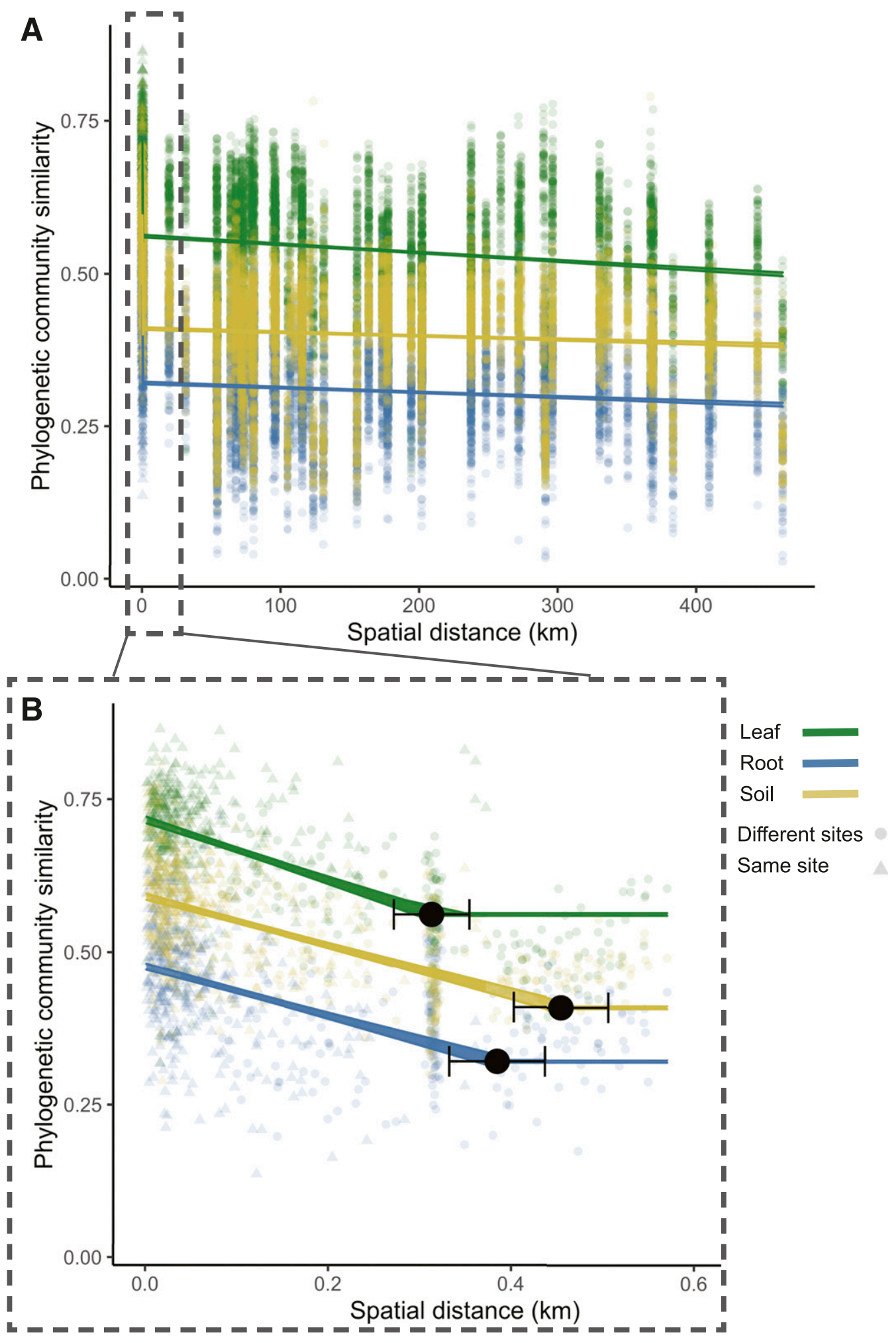

Fig. 2. Fitted piecewise regression models showing breakpoints in the relationship between pairwise phylogenetic community similarity and spatial distance for switchgrass leaf, root, and soil fungi. A, Distance-decay relationships across the full range of spatial distances. B, Subset of spatial distances focused on 0.27 to $0.49 \mathrm{~km}$, where breakpoints were identified. Breakpoints are indicated by black circles with $95 \%$ confidence intervals. See Supplementary Table S7 for model details. 
diverse fungal systems ranging from foliar endophytes in tropical grasses (Higgins et al. 2014) to ectomycorrhizas in pine trees (Peay et al. 2010). However, a subset of fungal ASVs in leaves tended to be more cosmopolitan than those in soils or roots, supporting the idea that at least some leaf fungi experience high dispersal rates. In addition, spatial variables were not included in any of the SEM models, suggesting that dispersal limitation is not the driver of these distance-decay patterns. Direct studies of
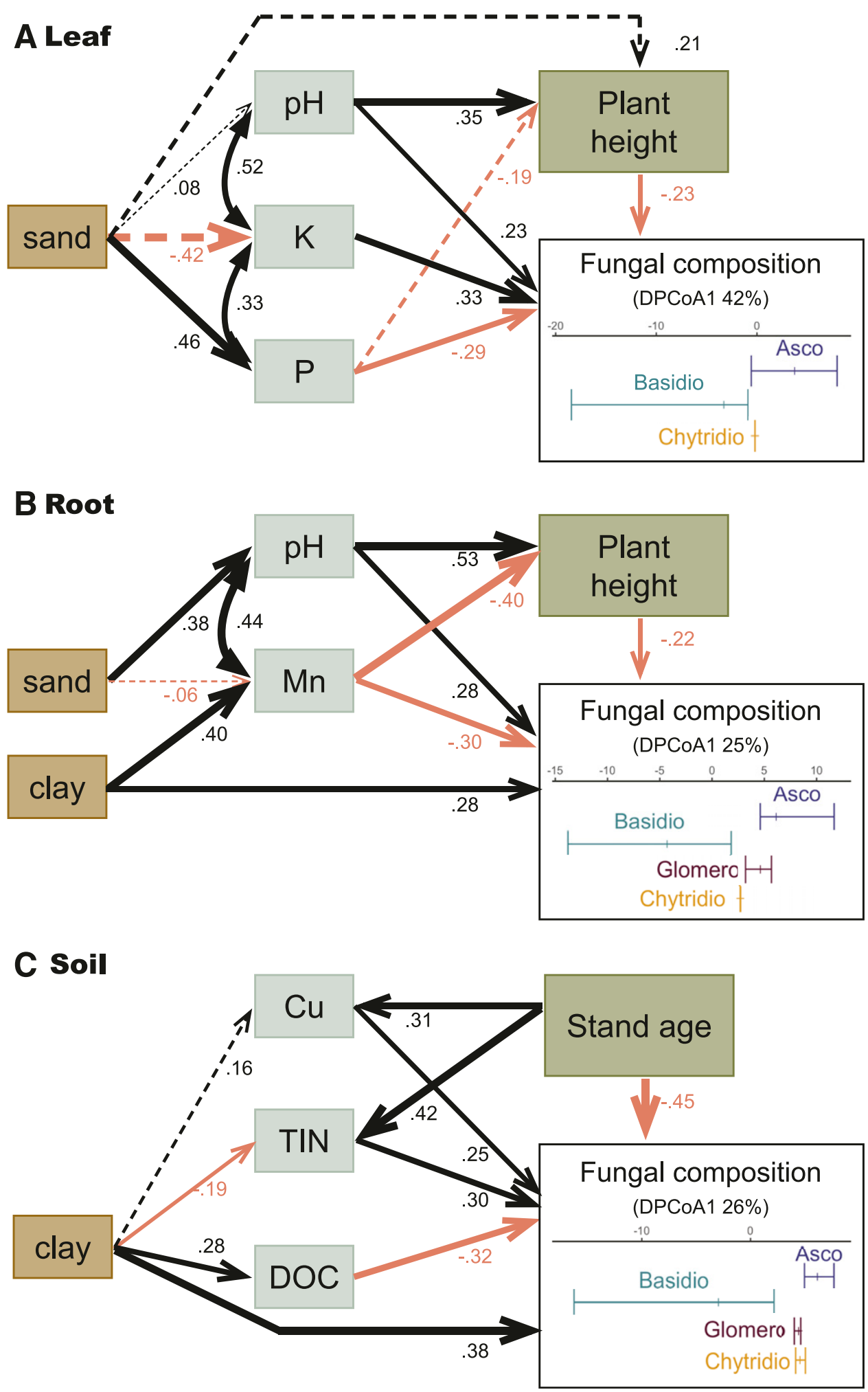

Fig. 3. Results of structural equation models for fungal composition (double principal coordinate analysis 1 [DPCoA1]) in A, leaf, B, root, and C, soil. Solid arrows indicate significant relationships $(P<0.05)$, dashed lines are nonsignificant, $\mathrm{TIN}=$ total inorganic $\mathrm{N}$, and DOC $=$ dissolved organic carbon. Arrow color indicates positive (black) or negative (red) slopes and thickness is proportional to standardized path coefficients. Standardized path coefficients are listed next to each arrow and can be interpreted to mean, for example in A, an increase in soil pH by 1 standard deviation (SD) from the mean results in an increase in plant height by $0.35 \mathrm{SD}$ from its mean. Variable units and transformations are in Supplementary Table S3. For DPCoA1, positions of dominant phyla (mean and range) are shown. 
airborne fungi at regional scales (Kivlin et al. 2014) and global estimates of fungal range sizes (Kivlin 2020) also find little dispersal limitation. Instead, it is likely that environmental heterogeneity and filtering is more likely responsible for observed patterns, and that heterogeneity occurs at similar scales for roots, leaves, and soils.
Soil properties cumulatively explained the most variation in fungal communities across all plant-associated habitats. In contrast to our hypotheses, this was true for leaves, where fungal communities were influenced by soil nutrients $\mathrm{K}$ and $\mathrm{P}$ as well as $\mathrm{pH}$. It is possible that leaf nutrients are tracking soil nutrient availability. For example, Kembel and Mueller (2014) found that leaf fungal

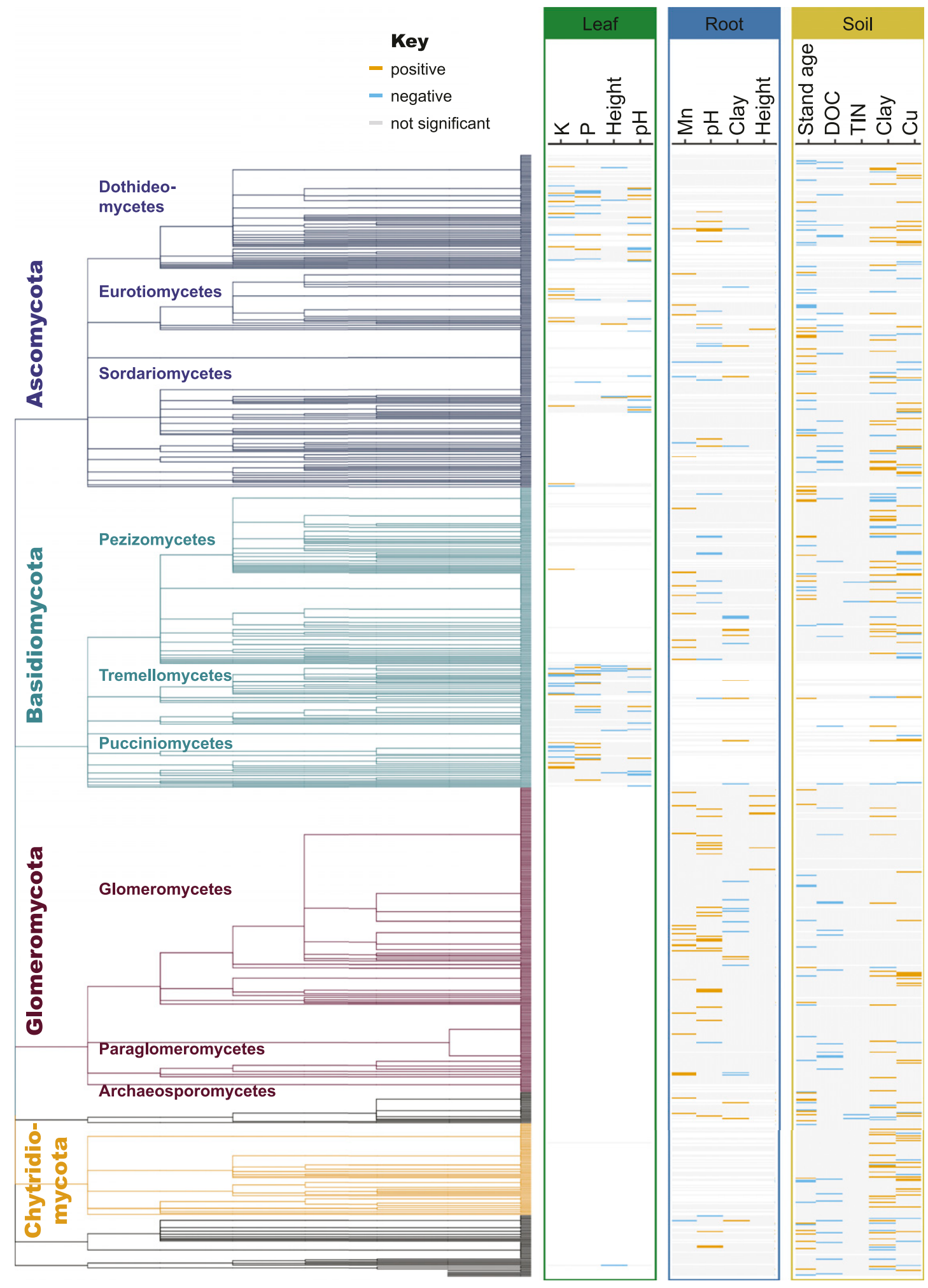

Fig. 4. Results of joint species distribution models for soil amplicon sequence variant (ASV) presence data showing significant relationships between ASVs and environmental variables. TIN = total inorganic $\mathrm{N}$ and DOC = dissolved organic carbon. The eight unlabeled phyla in black had few ASVs (<8\% combined). See Supplementary Table S11 for model fit and Supplementary Table S12 for significant coefficient estimates. 
community structure was correlated with leaf nutrients such as Al, $\mathrm{K}$, and $\mathrm{N}$ across tropical forest plant species. Similarly, when crop nutrient availability was experimentally altered with different fertilizer treatments, leaf fungi and bacteria were correlated with the treatments and tracked variation in specific leaf minerals (Darlison et al. 2019). Although the role of nutrients such as $\mathrm{K}$ in plant stomatal regulation, sugar transport, and enzymatic reactions is well known, how those processes might interact with foliar fungi remains to be discovered. Nutrients also played an important role in roots where increasing soil $\mathrm{Mn}$ resulted in more Rhizophagus mycorrhizal taxa, known to enhance foliar Mn and other elements in maize (Ramirez et al. 2019). However, higher Mn also favored saprotrophic Agaricomycetes fungi, which may reflect their capacity to produce $\mathrm{Mn}$ peroxidase enzymes that degrade lignin (Keiluweit et al. 2015).

In roots and soils, soil clay content was a key factor in explaining variation in fungal composition. The role of soil texture is consistent with other studies (de Vries et al. 2012) and likely reflects the physical and chemical challenges of resource acquisition in soils with different textures (Ritz and Young 2004). Among samples, clay content ranged from 2 to $45 \%$, and increasing clay was associated with increasing moisture, DOC, total $\mathrm{N}$, and other nutrients $(\mathrm{Mg}, \mathrm{Ca}, \mathrm{S}$, and $\mathrm{Cu}$ ) and decreasing total $\mathrm{P}$. Therefore, it is perhaps unsurprising to see a shift in the composition of Glomeromycota mycorrhizal fungi in higher clay. More interesting is the increase in serendipitoid mycorrhizas in roots, which can benefit switchgrass growth and germination and likely can access organic nutrients (Ghimire and Craven 2011; Ray et al. 2019). These patterns were also present in soil fungal communities with higher clay, reflecting the tight link between root and soil fungi.

Plant traits are often important controllers of inter- and intraspecific variation in host-associated microbiomes (DelgadoBaquerizo et al. 2018; Harrison et al. 2018; Kembel and Mueller 2014; Lumibao et al. 2020). Here, switchgrass height was a direct driver of both leaf and root fungi, with taller plants favoring Basidiomycetes. Plant size could represent the supply of carbon available to symbionts, as has been found for mycorrhizal fungi (Weremijewicz et al. 2016), or plant height may serve as an integrative measure of growing conditions that favor both plants and Basidiomycetes. This interpretation is supported by our finding in the SEMs that plant size mediates the role of soil $\mathrm{pH}$ and some nutrients on fungal communities in leaves and roots. Although plant height had a direct effect on fungi, it is also possible that we did not measure more proximal plant traits representing in-plant space and resource availability (i.e., specific leaf area, specific root length, and tissue nutrients) or susceptibility to colonization (i.e., puncture strength and defense compounds) that are good predictors of fungal communities across host plant species (González-Teuber et al. 2019; Kembel and Mueller 2014; Lumibao et al. 2020; Schröter et al. 2018). Similarly, we did not include information on switchgrass variety because it was not known at most sites; however, host genotype can be an important predictor in other grass crops (Deng et al. 2020; Sapkota et al. 2015). Additional studies will be needed to examine the potential role of other plant traits and their relationships to plant height.

In contrast to leaves and roots, soil fungi were primarily affected by the age of the switchgrass stand. In perennial agroecosystems such as switchgrass, stand age is often associated with enhanced soil C stocks (Bates et al. 2020; Ledo et al. 2020). In this study, however, older stands did not have greater soil $\mathrm{C}$ or organic matter but, instead, had plants with larger basal area and soils with more total and inorganic $\mathrm{P}$ and $\mathrm{Zn}$ and, to a lesser extent, soil inorganic $\mathrm{N}$ and $\mathrm{Cu}$. Likely due to the increased $\mathrm{P}$ and $\mathrm{N}$, older stands favored saprophytic Basidiomycota at the expense of mycorrhizal and endophytic fungi. These findings suggest that stand age effects on soils and their consequences for soil fungi are more complex than simple C increases. Our assessment of stand age is somewhat hampered, however, by the distribution of ages. The majority of stands ranged from 5 to 12 years in age, with only three sites planted less than 5 years prior and only two stands established 26 years prior (Supplementary Table S1). Including additional stands greater than 15 years since establishment might reveal different relationships.

Climate was not an important variable for explaining variation in fungal composition in this landscape, which contrasts with our expectations for foliar fungi based on previous work in switchgrass (Giauque and Hawkes 2013, 2016) and other plants (Barge et al. 2019; Würth et al. 2019). The lack of climate effects is likely due to limited climate gradients in North Carolina. For example, the maximum difference in mean annual precipitation was $183 \mathrm{~mm}$ across 14 sites, whereas studies with large rainfall effects have compared sites with $>400-\mathrm{mm}$ differences in annual precipitation (Giauque and Hawkes 2013). Much stronger gradients in soil texture and soil resource availability exist in this landscape due to geological events that formed three main ecoregions: the mountains with rocky, acidic soils; piedmont with clays; and coastal plain dominated by sandy soils (Horton and Zullo 1991).

Although we were able to identify that plant-associated habitat, soil properties, host size, and stand age partly explain switchgrass fungal communities, there remains a significant fraction of variability in these communities that is unexplained. The measure we used to represent fungal composition in SEMs (DPCoA1) explained $42 \%$ of variation in leaf fungal composition but only 25 and $26 \%$ of variation in root and soil communities, respectively; and, of that, predictors in the SEM explained 16 to $29 \%$ of variation in DPCoA1 scores. Joint species distribution models explained approximately 20 to $25 \%$ of variance in ASV abundances on average but performed much better at explaining the likelihood of ASV presences or absences based on reductions in root mean square error. This is similar to the majority of other studies of plant-associated fungi, which typically have 60 to $80 \%$ unexplained variation in fungal community structure (Cregger et al. 2018; Emery et al. 2017; Leff et al. 2018; Lumibao et al. 2020; Singer et al. 2019). Together, these findings suggest that unmeasured variables are influencing the outcome of fungal communities. It is possible that we did not measure relevant environmental variables, or that these were not measured on the scale that matters for microbes (Ladau and EloeFadrosh 2019). Other studies have found an important role of fungal species interactions (Maynard et al. 2018) and biotic interactions with other organisms such as bacteria or soil fauna (Anslan et al. 2018; Harrison et al. 2018). Identifying the critical variables that underpin niche-driven filtering in the fungal microbiome is a key unresolved issue in the field.

Although our understanding of microbial community assembly and distributions was initially dependent on a small number of bacterial studies (Martiny et al. 2006), fungi are increasingly being incorporated into biogeographic frameworks (Větrovský et al. 2019). Our findings that fungal community composition has distinct drivers among switchgrass leaves, roots, and soils highlights the diversity of environmental effects on assembly. These advances will help us to explain, predict, and manipulate microbial assemblages that support plant growth in managed and natural systems. For example, based on our findings, successful microbiome tools may not need to be explicitly scale dependent but, instead, would reflect key shared environmental conditions. Alteration of soil resources such as trace metals may also facilitate specific shifts in local microbial communities. In addition, based on the unique taxa in foliar systems, inoculation via direct aerial spray of foliar taxa onto newly opened leaves may be successful, whereas root 
microbes may require seed inoculation to overcome the close relationships to existing soil communities. The ecological mechanisms observed here open the door to future experiments that test the reliability of manipulating microbiomes in managed ecosystems. Other components of the phytobiome will likely also benefit from leveraging ecological mechanisms to optimize management.

\section{Acknowledgments}

We thank N. Yang, B. Whitaker, I. McBryde, J. Czel, E. Hardy, and M. Alvarez for assistance with labwork and fieldwork; North Carolina State University, North Carolina Department of Agriculture and Consumer Services, BASF Corp., Weyerhaeuser Co., S. Tian, C. Wilson, and E. Deal for providing site access; and J. Rocca, B. Whitaker, and R. Stuart for comments that improved the manuscript.

\section{LITERATURE CITED}

Anslan, S., Bahram, M., and Tedersoo, L. 2018. Seasonal and annual variation in fungal communities associated with epigeic springtails (Collembola spp.) in boreal forests. Soil Biol. Biochem. 116:245-252.

Bálint, M., Bartha, L., O’Hara, R. B., Olson, M. S., Otte, J., Pfenninger, M., Robertson, A. L., Tiffin, P., and Schmitt, I. 2014. Relocation, high-latitude warming and host genetic identity shape the foliar fungal microbiome of poplars. Mol. Ecol. 24:235-248.

Barge, E. G., Leopold, D. R., Peay, K. G., Newcombe, G., and Busby, P. E. 2019. Differentiating spatial from environmental effects on foliar fungal communities of Populus trichocarpa. J. Biogeogr. 46:2001-2011.

Bartlett, R. J., and Ross, D. S. 1988. Colorimetric determination of oxidizable carbon in acid soil solutions. Soil Sci. Soc. Am. J. 52:1191-1192.

Bates, C. T., Escalas, A., Kuang, J., Hale, L., Wang, Y., Herman, D., Nuccio, E.E., Wang, X., Fu, Y., Tian, R. and Wang, G. 2020. Conversion of marginal land into switchgrass conditionally accrues soil carbon and reduces methane consumption. bioRxiv. doi:10.1101/2020.03.18.997304

Bell, T. 2010. Experimental tests of the bacterial distance-decay relationship. ISME J. 4:1357-1365.

Bouyoucos, G. J. 1962. Hydrometer method improved for making particle size analyses of soils. Agron. J. 54:464-465.

Bryant, J. A., Lamanna, C., Morlon, H., Kerkhoff, A. J., Enquist, B. J., and Green, J. L. 2008. Microbes on mountainsides: Contrasting elevational patterns of bacterial and plant diversity. Proc. Natl. Acad. Sci. U.S.A. 105:11505-11511.

Büchi, L., and Vuilleumier, S. 2014. Coexistence of specialist and generalist species is shaped by dispersal and environmental factors. Am. Nat. 183:612-624.

Callahan, B. J., McMurdie, P. J., Rosen, M. J., Han, A. W., Johnson, A. J. A., and Holmes, S. P. 2016. DADA2: High-resolution sample inference from Illumina amplicon data. Nat. Methods 13:581-583.

Chaudhary, V. B., Nolimal, S., Sosa Hernández, M. A., Egan, C., and Kastens, J. 2020. Trait-based aerial dispersal of arbuscular mycorrhizal fungi. New Phytol. 228:238-252.

Chesson, P. 2000. Mechanisms of maintenance of species diversity. Annu. Rev. Ecol. Syst. 31:343-366.

Cline, L. C., and Zak, D. R. 2013. Dispersal limitation structures fungal community assembly in a long-term glacial chronosequence. Environ. Microbiol. 16:1538-1548.

Collyer, M. L., and Adams, D. C. 2018. RRPP: An R package for fitting linear models to high-dimensional data using residual randomization. Methods Ecol. Evol. 9:1772-1779.

Craine, J. M., Lee, W. G., Bond, W. J., Williams, R. J., and Johnson, L. C. 2005. Environmental constraints on a global relationship among leaf and root traits of grasses. Ecology 86:12-19.

Cregger, M. A., Veach, A. M., Yang, Z. K., Crouch, M. J., Vilgalys, R., Tuskan, G. A., and Schadt, C. W. 2018. The Populus holobiont: Dissecting the effects of plant niches and genotype on the microbiome. Microbiome 6:31.

Darlison, J., Mogren, L., Rosberg, A. K., Grudén, M., Minet, A., Liné, C., Mieli, M., Bengtsson, T., Håkansson, Å., Uhlig, E., and Becher, P. G. 2019. Leaf mineral content govern microbial community structure in the phyllosphere of spinach (Spinacia oleracea) and rocket (Diplotaxis tenuifolia). Sci. Total Environ. 675:501-512

Davies, R. B. 2002. Hypothesis testing when a nuisance parameter is present only under the alternative: Linear model case. Biometrika 89:484-489.
Delgado-Baquerizo, M., Fry, E. L., Eldridge, D. J., de Vries, F. T., Manning, P., Hamonts, K., Kattge, J., Boenisch, G., Singh, B. K., and Bardgett, R. D. 2018. Plant attributes explain the distribution of soil microbial communities in two contrasting regions of the globe. New Phytol. 219:574-587.

Deng, S., Caddell, D., Yang, J., Dahlen, L., Washington, L., and Coleman-Derr, D. 2020. Genome wide association study reveals plant loci controlling heritability of the rhizosphere microbiome. bioRxiv. doi:10.1101/2020.02.21.960377

Deshpande, V., Wang, Q., Greenfield, P., Charleston, M., Porras-Alfaro, A., Kuske, C. R., Cole, J. R., Midgley, D. J., and Tran-Dinh, N. 2017. Fungal identification using a Bayesian classifier and the Warcup training set of internal transcribed spacer sequences. Mycologia 108:1-5.

de Vries, F. T., Manning, P., Tallowin, J. R. B., Mortimer, S. R., Pilgrim, E. S., Harrison, K. A., Hobbs, P. J., Quirk, H., Shipley, B., Cornelissen, J. H., and Kattge, J. 2012. Abiotic drivers and plant traits explain landscape-scale patterns in soil microbial communities. Ecol. Lett. 15:1230-1239.

Doane, T. A., and Horwáth, W. R. 2003. Spectrophotometric determination of nitrate with a single reagent. Anal. Lett. 36:2713-2722.

Emery, S. M., Reid, M. L., Bell-Dereske, L., and Gross, K. L. 2017. Soil mycorrhizal and nematode diversity vary in response to bioenergy crop identity and fertilization. Glob. Change Biol. Bioenergy 9:1644-1656.

Fan, K., Cardona, C., Li, Y., Shi, Y., Xiang, X., Shen, C., Wang, H., Gilbert, J. A., and Chu, H. 2017. Rhizosphere-associated bacterial network structure and spatial distribution differ significantly from bulk soil in wheat crop fields. Soil Biol. Biochem. 113:275-284.

Friedman, J., Hastie, T., and Tibshirani, R. 2010. Regularization paths for generalized linear models via coordinate descent. J. Stat. Softw. 33:1-22.

Frøslev, T. G., Kjøller, R., Bruun, H. H., Ejrnæs, R., Brunbjerg, A. K., Pietroni, C., and Hansen, A. J. 2017. Algorithm for post-clustering curation of DNA amplicon data yields reliable biodiversity estimates. Nat. Commun. 8:1188.

Gao, C., Montoya, L., Xu, L., Madera, M., Hollingsworth, J., Purdom, E., Singan, V., Vogel, J., Hutmacher, R. B., Dahlberg, J. A., and Coleman-Derr, D. 2020. Fungal community assembly in drought-stressed sorghum shows stochasticity, selection, and universal ecological dynamics. Nat. Commum. 11:34.

Ghimire, S. R., Charlton, N. D., Bell, J. D., Krishnamurthy, Y. L., and Craven, K. D. 2010. Biodiversity of fungal endophyte communities inhabiting switchgrass (Panicum virgatum L.) growing in the native tallgrass prairie of northern Oklahoma. Fungal Divers. 47:19-27.

Ghimire, S. R., and Craven, K. D. 2011. Enhancement of switchgrass (Panicum virgatum $L$.) biomass production under drought conditions by the ectomycorrhizal fungus Sebacina vermifera. J. Appl. Environ. Microbiol. 77:7063-7067.

Giauque, H., and Hawkes, C. V. 2013. Climate affects symbiotic fungal endophyte diversity and performance. Am. J. Bot. 100:1435-1444.

Giauque, H., and Hawkes, C. V. 2016. Historical and current climate drive spatial and temporal patterns in fungal endophyte diversity. Fungal Ecol. 20:108-114.

González-Teuber, M., Vilo, C., Guevara-Araya, M. J., Salgado-Luarte, C., and Gianoli, E. 2019. Leaf resistance traits influence endophytic fungi colonization and community composition in a South American temperate rainforest. J. Ecol. 108:1019-1029.

Grady, K. L., Sorensen, J. W., Stopnisek, N., Guittar, J., and Shade, A. 2019. Assembly and seasonality of core phyllosphere microbiota on perennial biofuel crops. Nat. Commun. 10:4135.

Gusareva, E. S., Acerbi, E., Lau, K. J. X., Luhung, I., Premkrishnan, B. N. V., Kolundžija, S., Purbojati, R. W., Wong, A., Houghton, J. N., Miller, D., and Gaultier, N. E. 2019. Microbial communities in the tropical air ecosystem follow a precise diel cycle. Proc. Natl. Acad. Sci. U.S.A. 116:23299-23308.

Hanson, C. A., Fuhrman, J. A., Horner-Devine, M. C., and Martiny, J. B. H. 2012. Beyond biogeographic patterns: Processes shaping the microbial landscape. Nature 10:497-506.

Harrison, J. G., Parchman, T. L., Cook, D., Gardner, D. R., and Forister, M. L. 2018. A heritable symbiont and host-associated factors shape fungal endophyte communities across spatial scales. J. Ecol. 106:2274-2286.

Higgins, K. L., Arnold, A. E., Coley, P. D., and Kursar, T. A. 2014. Communities of fungal endophytes in tropical forest grasses: Highly diverse host- and habitat generalists characterized by strong spatial structure. Fungal Ecol. 8:1-11.

Horton, J. W., Jr., and Zullo, V. A., eds. 1991. The Geology of the Carolinas. University of Tennessee Press, Knoxville, TN, U.S.A.

Keiluweit, M., Nico, P., Harmon, M. E., Mao, J., Pett-Ridge, J., and Kleber, M. 2015. Long-term litter decomposition controlled by manganese redox cycling. Proc. Natl. Acad. Sci. U.S.A. 112:E5253-E5260.

Kembel, S. W., Cowan, P. D., Helmus, M. R., Cornwell, W. K., Morlon, H., Ackerly, D. D., Blomberg, S. P., and Webb, C. O. 2010. Picante: R tools for integrating phylogenies and ecology. Bioinformatics 26:1463-1464.

Kembel, S. W., and Mueller, R. C. 2014. Plant traits and taxonomy drive host associations in tropical phyllosphere fungal communities. Botany 92:303-311. 
Kivlin, S. N. 2020. Global mycorrhizal fungal range sizes vary within and among mycorrhizal guilds but are not correlated with dispersal traits. J. Biogeogr. 47: 1994-2001.

Kivlin, S. N., Winston, G. C., Goulden, M. L., and Treseder, K. K. 2014. Environmental filtering affects soil fungal community composition more than dispersal limitation at regional scales. Fungal Ecol. 12:14-25.

Kleczewski, N. M., Bauer, J. T., Bever, J. D., Clay, K., and Reynolds, H. L. 2012. A survey of endophytic fungi of switchgrass (Panicum virgatum) in the Midwest, and their putative roles in plant growth. Fungal Ecol. 5:521-529.

Ladau, J., and Eloe-Fadrosh, E. A. 2019. Spatial, temporal, and phylogenetic scales of microbial ecology. Trends Microbiol. 27:662-669.

Ledo, A., Smith, P., Zerihun, A., Whitaker, J., Vicente-Vicente, J. L., Qin, Z., McNamara, N. P., Zinn, Y. L., Llorente, M., Liebig, M., and Kuhnert, M. 2020. Changes in soil organic carbon under perennial crops. Glob. Change Biol. 26:4158-4168.

Leff, J. W., Bardgett, R. D., Wilkinson, A., Jackson, B. G., Pritchard, W. J., De Long, J. R., Oakley, S., Mason, K. E., Ostle, N. J., Johnson, D., Baggs, E. M., and Fierer, N. 2018. Predicting the structure of soil communities from plant community taxonomy, phylogeny, and traits. ISME J. 12:1794-1805.

Lindahl, B. D., and Tunlid, A. 2014. Ectomycorrhizal fungi - potential organic matter decomposers, yet not saprotrophs. New Phytol. 205:1443-1447.

Lumibao, C. Y., Bernik, B. M., Formel, S. K., Kandalepas, D., Mighell, K. L., Pardue, J., Van Bael, S. A., and Blum, M. J. 2020. Rhizosphere microbial communities reflect genotypic and trait variation in a salt marsh ecosystem engineer. Am. J. Bot. 107:941-949.

Mamet, S. D., Lamb, E. G., Piper, C. L., Winsley, T., and Siciliano, S. D. 2017. Archaea and bacteria mediate the effects of native species root loss on fungi during plant invasion. ISME J. 11:1261-1275.

Martins, F., Pereira, J. A., Bota, P., Bento, A., and Baptista, P. 2016. Fungal endophyte communities in above- and belowground olive tree organs and the effect of season and geographic location on their structures. Fungal Ecol. 20: 193-201.

Martiny, J. B. H., Bohannan, B. J. M., Brown, J. H., Colwell, R. K., Fuhrman, J. A., Green, J. L., Horner-Devine, M. C., Kane, M., Krumins, J. A., Kuske, C. R., and Morin, P. J. 2006. Microbial biogeography: Putting microorganisms on the map. Nat. Rev. Microbiol. 4:102-112.

Maynard, D. S., Covey, K. R., Crowther, T. W., Sokol, N. W., Morrison, E. W., Frey, S. D., Van Diepen, L. T., and Bradford, M. A. 2018. Species associations overwhelm abiotic conditions to dictate the structure and function of wood-decay fungal communities. Ecology 99:801-811.

McCutcheon, J. P., and Lekberg, Y. 2019. Symbiosis: Fungi as shrewd trade negotiators. Curr. Biol. 29:R570-R572.

Muggeo, V. M. R. 2003. Estimating regression models with unknown breakpoints. Stat. Med. 22:3055-3071.

Mulvaney, R. L. 1996. Nitrogen-Inorganic forms. Pages 1123-1184 in: Methods of Soil Analysis: Part 3 Chemical Methods. D. L. Sparks, A. L. Page, P. A. Helmke, R. H. Loeppert, P. N. Soltanpour, M. A. Tabatabai, C. T. Johnston, and M. E. Sumner, eds. SSSA Book Series. Soil Science Society of America, Madison, WI, U.S.A.

Nilsson, R. H., Larsson, K.-H., Taylor, A. F. S., Bengtsson-Palme, J., Jeppesen, T. S., Schigel, D., Kennedy, P., Picard, K., Glöckner, F. O., Tedersoo, L., Saar, I., Kõljalg, U., and Abarenkov, K. 2018. The UNITE database for molecular identification of fungi: Handling dark taxa and parallel taxonomic classifications. Nucleic Acids Res. 47:D259-D264.

Pavoine, S., Dufour, A.-B. A.-B., and Chessel, D. 2004. From dissimilarities among species to dissimilarities among communities: A double principal coordinate analysis. J. Theor. Biol. 228:523-537.

Peay, K. G., Garbelotto, M., and Bruns, T. D. 2010. Evidence of dispersal limitation in soil microorganisms: Isolation reduces species richness on mycorrhizal tree islands. Ecology 91:3631-3640.

Qian, X., Li, H., Wang, Y., Wu, B., Wu, M., Chen, L., Li, X., Zhang, Y., Wang, X., Shi, M., and Zheng, Y. 2019. Leaf and root endospheres harbor lower fungal diversity and less complex fungal co-occurrence patterns than rhizosphere. Front. Microbiol. 10:1015.

Queloz, V., Sieber, T. N., Holdenrieder, O., McDonald, B. A., and Grünig, C. R. 2010. No biogeographical pattern for a root-associated fungal species complex. Glob. Ecol. Biogeogr. 20:160-169.

Ramirez, K. S., Snoek, L. B., Koorem, K., Geisen, S., Bloem, L. J., ten Hooven, F., Kostenko, O., Krigas, N., Manrubia, M., Caković, D., van Raaij, D., Tsiafouli, M. A., Vreš, B., Čelik, T., Weser, C., Wilschut, R. A., and van der Putten, W. H. 2019. Range-expansion effects on the belowground plant microbiome. Nat. Ecol. Evol. 3:604-611.

Ray, P., Abraham, P. E., Guo, Y., Giannone, R. J., Engle, N. L., Yang, Z. K., Jacobson, D., Hettich, R. L., Tschaplinski, T. J., and Craven, K. D. 2019.
Scavenging organic nitrogen and remodelling lipid metabolism are key survival strategies adopted by the endophytic fungi, Serendipita vermifera and Serendipita bescii to alleviate nitrogen and phosphorous starvation in vitro. Environ. Microbiol. Rep. 11:548-557.

R Core Team. 2014. R: A Language and Environment for Statistical Computing, 3rd ed. R Foundation for Statistical Computing, Vienna, Austria. http://www.R-project.org/

Ritz, K., and Young, I. M. 2004. Interactions between soil structure and fungi. Mycologist 18:52-59.

Robert, V., Vu, D., Amor, A. B. H., van de Wiele, N., Brouwer, C., Jabas, B., Szoke, S., Dridi, A., Triki, M., Ben Daoud, S., and Chouchen, O. 2013. MycoBank gearing up for new horizons. IMA Fungus 4:371-379.

Robertson, G. P., Coleman, D. C., Bledsoe, C. S., and Sollins, P., eds. 1999. Standard Soil Methods for Long-term Ecological Research. Oxford University Press, New York, NY, U.S.A.

Rosseel, Y. 2012. lavaan: An R package for structural equation modeling. J. Stat. Softw. 48:1-36.

Sánchez Márquez, S., Bills, G. F., Domínguez Acuña, L., and Zabalgogeazcoa, I. 2010. Endophytic mycobiota of leaves and roots of the grass Holcus lanatus. Fungal Divers. 41:115-123.

Sapkota, R., Knorr, K., Jørgensen, L. N., O’Hanlon, K. A., and Nicolaisen, M. 2015. Host genotype is an important determinant of the cereal phyllosphere mycobiome. New Phytol. 207:1134-1144.

Schröter, K., Wemheuer, B., Pena, R., Schöning, I., Ehbrecht, M., Schall, P., Ammer, C., Daniel, R., and Polle, A. 2018. Assembly processes of trophic guilds in the root mycobiome of temperate forests. Mol. Ecol. 28:348-364.

Scott-Denton, L. E., Rosenstiel, T. N., and Monson, R. K. 2006. Differential controls by climate and substrate over the heterotrophic and rhizospheric components of soil respiration. Glob. Change Biol. 12:205-216.

Singer, E., Bonnette, J., Kenaley, S. C., Woyke, T., and Juenger, T. E. 2019. Plant compartment and genetic variation drive microbiome composition in switchgrass roots. Environ. Microbiol. Rep. 11:185-195.

Taylor, D. L., Walters, W. A., Lennon, N. J., Bochicchio, J., Krohn, A., Caporaso, J. G., and Pennanen, T. 2016. Accurate estimation of fungal diversity and abundance through improved lineage-specific primers optimized for Illumina amplicon sequencing. Appl. Environ. Microbiol. 82: 7217-7226.

Tedersoo, L., Sánchez-Ramírez, S., Kõljalg, U., Bahram, M., Döring, M., Schigel, D., May, T., Ryberg, M., and Abarenkov, K. 2018. High-level classification of the Fungi and a tool for evolutionary ecological analyses. Fungal Divers. 90:135-159.

Thomas, D. C., Vandegrift, R., Ludden, A., Carroll, G. C., and Roy, B. A. 2016. Spatial ecology of the fungal genus Xylaria in a tropical cloud forest. Biotropica 48:381-393.

Tikhonov, G., Opedal, Ø. H., Abrego, N., Lehikoinen, A., de Jonge, M. M. J., Oksanen, J., and Ovaskainen, O. 2020. Joint species distribution modelling with the R-package HMSC. Methods Ecol. Evol. 11:442-447.

Tilman, D. 2004. Niche tradeoffs, neutrality, and community structure: A stochastic theory of resource competition, invasion, and community assembly. Proc. Natl. Acad. Sci. U.S.A. 101:10854-10861.

Vacher, C., Cordier, T., and Vallance, J. 2016. Phyllosphere fungal communities differentiate more thoroughly than bacterial communities along an elevation gradient. Microb. Ecol. 72:1-3.

Vance, E. D., Brookes, P. C., and Jenkinson, D. S. 1987. An extraction method for measuring soil microbial biomass C. Soil Biol. Biochem. 19:703-707.

Van der Heijden, M. G. A. 2004. Arbuscular mycorrhizal fungi as support systems for seedling establishment in grassland. Ecol. Lett. 7:293-303.

Větrovský, T., Kohout, P., Kopecký, M., Machac, A., Man, M., Bahnmann, B. D., Brabcová, V., Choi, J., Meszárošová, L., Human, Z. R., and Lepinay, C. 2019. A meta-analysis of global fungal distribution reveals climate-driven patterns. Nat. Commun. 10:5142.

Vos, M., Wolf, A. B., Jennings, S. J., and Kowalchuk, G. A. 2013. Micro-scale determinants of bacterial diversity in soil. FEMS Microbiol. Rev. 37:936-954.

Wang, J., Pan, F., Soininen, J., Heino, J., and Shen, J. 2016. Nutrient enrichment modifies temperature-biodiversity relationships in large-scale field experiments. Nat. Commun. 7:13960.

Weremijewicz, J., Sternberg, L. D. S. L. O., and Janos, D. P. 2016. Common mycorrhizal networks amplify competition by preferential mineral nutrient allocation to large host plants. New Phytol. 212:461-471.

Würth, D. G., Dahl, M. B., Trouillier, M., Wilmking, M., Unterseher, M., Scholler, M., Sørensen, S., Mortensen, M., and Schnittler, M. 2019. The needle mycobiome of Picea glauca-A dynamic system reflecting surrounding environment and tree phenological traits. Fungal Ecol. 41:177-186.

Zhou, J., and Ning, D. 2017. Stochastic Community assembly: Does it matter in microbial ecology? Microbiol. Mol. Biol. Rev. 81:e00002-17. 